Ugail H (2004): "Spine Based Shape Parameterisaion for PDE Surfaces," Journal of Computing, 72 195--206. 


\title{
Spine Based Shape Parameterisation for PDE Surfaces
}

\author{
Hassan Ugail \\ Department of Electronic Imaging and Media Communications \\ School of Informatics, University of Bradford \\ Bradford BD7 1DP, UK \\ h.ugail@bradford.ac.uk
}

\begin{abstract}
The aim of this paper is to show how the spine of a PDE surface can be generated and how it can be used to efficiently parameterise a PDE surface. For the purpose of the work presented here an approximate analytic solution form for the chosen PDE is utilised. It is shown that the spine of the PDE surface is then computed as a by-product of this analytic solution. Furthermore, it is shown that a parameterisation can be introduced on the spine enabling intuitive manipulation of PDE surfaces.
\end{abstract}

\section{Introduction}

An important requirement for modern CAD systems is the ability to be able to parameterise the shapes of complex objects with ease. In parametric design the basic approach adopted is to develop a generic description of an object or a series of objects to which a given set of design parameters can be associated. Shape manipulation (usually in an interactive environment) can then be carried out by choosing appropriate values for the chosen design parameters. To this end it becomes essential that the chosen parameters not only describe the shape intuitively but also enable the designer to manipulate the shape with ease.

Presently there exist many commercial CAD systems which employ the conventional polynomial surface modelling schemes. A fine example of the polynomial based surface modelling scheme is the Non-Uniform Rational BSplines (NURBS) [8]. NURBS are based on simple polynomial functions to which weights and knots collectively known as control points are associated. These control points are often identified as the design parameters. For simple shapes the interactive manipulation through changes in the position of the control points become intuitive and somewhat predictable. However, as the design becomes more complex the process of shape manipulation, by way of manipulating the control points, becomes time-consuming and rather nonintuitive. 
PDE surfaces have recently emerged as a powerful shape modelling technique $[5,6,11,12]$. It has been demonstrated how a designer sitting in front of a workstation is able to create and manipulate complex geometry interactively in real time [11]. Furthermore, it has been shown that complex geometry can be efficiently parameterised both for intuitive shape manipulation [12] and for efficient design optimisation [2].

Previous work on shape parameterisation allows a designer to choose shape parameters on the boundary curves that define the PDE surface [11]. It has been shown that intuitive shape manipulation can then be performed by means of changing these parameters. Although in this earlier work it has been demonstrated that the manipulations of complex surface shapes by means of manipulating the corresponding boundary curves is intuitive, for complex objects that are built from a number of multiple PDE surface patches, it has been noted that in certain circumstances the process of shape manipulation can be time-consuming. Therefore, the motivation behind this work is to introduce new techniques that allow a designer to manipulate shapes of complex objects with less effort and to further parameterise the object in an intuitive fashion. For this purpose the basic idea used here is to exploit the use of a skeleton or a 'spine' associated with the PDE surfaces.

Generally speaking the spine of an object is described to be a curve in 3space that follows the 'centreline' of that object. Thus, the spine of an object has a close geometric resemblance to the more widely known shape entity known as the medial axis or the skeleton [3, 7]. Bearing this in mind, one could therefore imagine that the spine of a shape brings out the symmetries in that shape. It can also be noted that the spine in general has far richer topologies than the shape it is derived from. Apart from the rich geometric properties the spine posses, its intuitive appeal in applications in geometric manipulations can also be noted. For example, many authors have stated the flexibility of the medial axis and its ability to naturally capture important shape characteristics of an object $[10,9,4]$. Since the spine of an object has a close resemblance to the medial axis it can be seen that the spine of an object can therefore naturally characterise the shape from which it is derived from.

The aim of this paper is to show how the spine of a PDE surface can be created and utilised in order to characterise PDE surfaces as well as to enable the development of further intuitive techniques for powerful shape parameterisation. By exploiting the structural form of a closed form solution for the chosen PDE it is shown how the spine of a PDE surface can be generated as a by-product of this solution. Furthermore, it is shown that the spine of the PDE surface patch is represented as a parametric polynomial function that can be used as a shape manipulation tool to deform the shape 
in an intuitive fashion. To demonstrate the ideas presented here, practical examples of shapes involving PDE surfaces are discussed throughout the paper.

\section{PDE Surfaces}

A PDE surface is a parametric surface patch $\underline{X}(u, v)$, defined as a function of two parameters $u$ and $v$ on a finite domain $\Omega \subset R^{2}$, by specifying boundary data around the edge region of $\partial \Omega$. Typically the boundary data are specified in the form of $\underline{X}(u, v)$ and a number of its derivatives on $\partial \Omega$. Moreover, this approach regards the coordinates of the $(u, v)$ point as a mapping from that point in $\Omega$ to a point in the physical space. To satisfy these requirements the surface $\underline{X}(u, v)$ is regarded as a solution of a PDE based on the bi-harmonic equation $\nabla^{4}=0$ namely,

$$
\left(\frac{\partial^{2}}{\partial u^{2}}+a^{2} \frac{\partial^{2}}{\partial v^{2}}\right)^{2} \underline{X}(u, v)=0 .
$$

Here the boundary conditions on the function $\underline{X}(u, v)$ and its normal derivatives $\frac{\partial \underline{X}}{\partial n}$ are imposed at the edges of the surface patch. The parameter $a$ is a special design parameter which controls the relative smoothing of the surface in the $u$ and $v$ directions [12].

\subsection{Solution of the PDE}

There exist many methods to determine the solution of Equation (1) ranging from analytic solution techniques to sophisticated numerical methods. For the work described here restricting to periodic boundary conditions a closed form analytic solution of Equation (1) is utilised.

Choosing the parametric region to be $0 \leq u \leq 1$ and $0 \leq v \leq 2 \pi$, the periodic boundary conditions can be expressed as, $\underline{X}(0, v)=\underline{P}_{1}(v)$, $\underline{X}(1, v)=\underline{P}_{2}(v), \underline{X}_{u}(0, v)=\underline{d}_{1}(v)$, and $\underline{X}_{u}(1, v)=\underline{d}_{2}(v)$.

Note that the boundary conditions $\underline{P}_{0}(v)$ and $\underline{P}_{1}(v)$ define the edges of the surface patch at $u=0$ and $u=1$ respectively. Using the method of separation of variables, the analytic solution of Equation (1) can be written as,

$$
\underline{X}(u, v)=\underline{A}_{0}(u)+\sum_{n=1}^{\infty}\left[\underline{A}_{n}(u) \cos (n v)+\underline{B}_{n}(u) \sin (n v)\right],
$$

where

$$
\underline{A}_{0}=\underline{a}_{00}+\underline{a}_{01} u+\underline{a}_{02} u^{2}+\underline{a}_{03} u^{3},
$$




$$
\begin{aligned}
& \underline{A}_{n}=\underline{a}_{n 1} e^{a n u}+\underline{a}_{n 2} u e^{a n u}+\underline{a}_{n 3} e^{-a n u}+\underline{a}_{n 4} u e^{-a n u}, \\
& \underline{B}_{n}=\underline{b}_{n 1} e^{a n u}+\underline{b}_{n 2} u e^{a n u}+\underline{b}_{n 3} e^{-a n u}+\underline{b}_{n 4} u e^{-a n u},
\end{aligned}
$$

where $\underline{a}_{00}, \underline{a}_{01}, \underline{a}_{02}, \underline{a}_{03} \underline{a}_{n 1}, \underline{a}_{n 2}, \underline{a}_{n 3}, \underline{a}_{n 4}, \underline{b}_{n 1} \underline{b}_{n 2}, \underline{b}_{n 3}$ and $\underline{b}_{n 4}$ are vector constants, whose values are determined by the imposed boundary conditions at $u=0$ and $u=1$.

For a general set of boundary conditions, in order to define the various constants in the solution, it is necessary to Fourier analyse the boundary conditions and identify the various Fourier coefficients. Where the boundary conditions can be expressed exactly in terms of a finite Fourier series, the solution given in Equation (2) will also be finite. However, this is often not possible, in which case the solution will be the infinite series given Equation (2).

The technique for finding an approximation to $\underline{X}(u, v)$ is based on the sum of the first few Fourier modes and a 'remainder term', i.e.,

$$
\underline{X}(u, v) \simeq \underline{A}_{0}(u)+\sum_{n=1}^{N}\left[\underline{A}_{n}(u) \cos (n v)+\underline{B}_{n}(u) \sin (n v)\right]+\underline{R}(u, v),
$$

where $N$ is usually small (e.g. $N<10$ ) and $\underline{R}(u, v)$ is a remainder function defined as,

$$
\underline{R}(u, v)=\underline{r}_{1}(v) e^{w u}+\underline{r}_{2}(v) e^{w u}+\underline{r}_{3}(v) e^{-w u}+\underline{r}_{4}(v) e^{-w u},
$$

where $\underline{r}_{1}, \underline{r}_{2}, \underline{r}_{3}, \underline{r}_{4}$ and $w$ are obtained by considering the difference between the original boundary conditions and the boundary conditions satisfied by the function,

$$
\underline{F}(u, v)=\underline{A}_{0}(u)+\sum_{n=1}^{N}\left[\underline{A}_{n}(u) \cos (n v)+\underline{B}_{n}(u) \sin (n v)\right] .
$$

An important point to note here is that although the solution is approximate this new solution technique guarantees that the chosen boundary conditions are exactly satisfied since the remainder function $\underline{R}(u, v)$ is calculated by means of the difference between the original boundary conditions and the boundary conditions satisfied by the function $\underline{F}(u, v)[1]$.

Fig. 1 demonstrates the interactive design of a typical PDE surface where the boundary conditions for the PDE are chosen in the form of curves in 3-space shown in Fig. 1(a). The corresponding PDE surface shape is shown in Fig. 1(b). Here the curves marked $P_{1}$ and $P_{2}$ correspond to the boundary conditions on the function $\underline{X}(u, v)$, where $P_{1}(v)=\underline{X}(0, v)$ and $P_{2}(v)=\underline{X}(1, v)$. A vector field corresponding to the difference between 


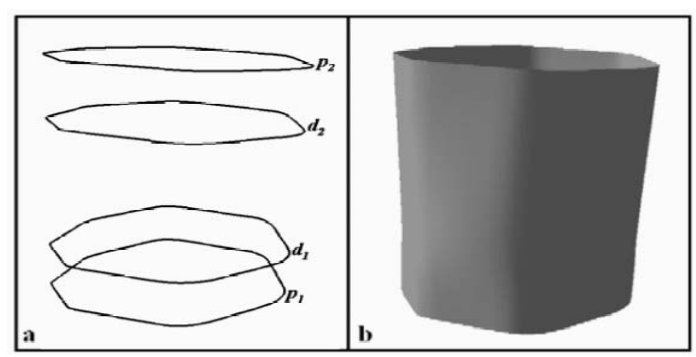

Figure 1: A typical PDE surface created interactively. (a) The boundary conditions defined in the form of curves in 3-space. (b) The resulting surface shape.

the points on the curves marked $P_{1}$ and $P_{2}$ and those marked $d_{1}$ and $d_{2}$ respectively, corresponds to the conditions on the function $\frac{\partial X}{\partial n}$ such that $\frac{\partial \underline{X}}{\partial n}=[\underline{p}(v)-\underline{d}(v)] s$, where $s$ is a scalar.

It can be appreciated that from a design point of view this boundary curve based approach is a user-friendly tool for PDE surface manipulation in an interactive environment. Previous work on interactive design using the PDE method demonstrates these techniques [11].

\subsection{The Spine of a PDE surface}

Taking the form of Equation (2) one could observe the following properties of the analytic solution that allows us to extract the spine of a PDE surface as a by-product of the solution.

Firstly the term $\underline{A}_{0}$ in Equation (2) is a cubic polynomial of the parameter $u$. Secondly it can be seen that for each point $\underline{X}(u, v)$ on the surfaces the term $\sum_{n=1}^{\infty}\left[\underline{A}_{n}(u) \cos (n v)+\underline{B}_{n}(u) \sin (n v)\right]$ in Equation (2) describes the radial position of the point $\underline{X}(u, v)$ away from a point at $\underline{A}_{0}$. Finally applying the triangle inequality to Equation (2) one can see that,

$$
\left\|\underline{A}_{0}(u)\right\| \leq\|\underline{X}(u, v)\|-\left\|\sum_{n=1}^{\infty}\left[\underline{A}_{n}(u) \cos (n v)+\underline{B}_{n}(u) \sin (n v)\right]\right\| .
$$

Thus, the term $\underline{A}_{0}$ which is a cubic polynomial of the parameter $u$ traces out a curve in 3-space which follows the 'centreline' of the surfaces patch. Therefore, using the solution technique described in Equation (2) a surface point $\underline{X}(u, v)$ may be regarded as being composed of sum of a vector $\underline{A}_{0}$ giving the position on the spine of the surface and a radius vector defined by the term $\sum_{n=1}^{\infty}\left[\underline{A}_{n}(u) \cos (n v)+\underline{B}_{n}(u) \sin (n v)\right]$ providing the position of $\underline{X}(u, v)$ relative to the spine. More precisely one could state that for a PDE 


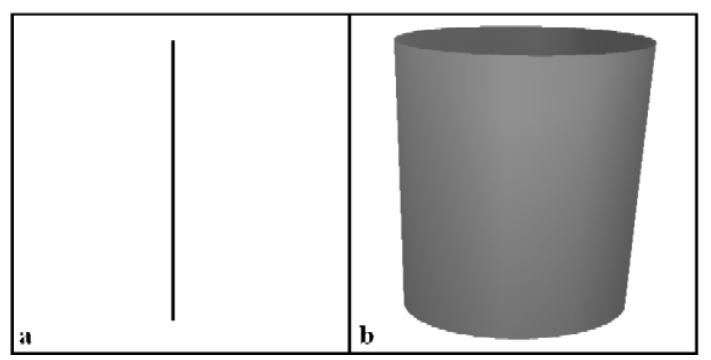

Figure 2: Description of the spine of a PDE surface. (a) The spine described by the $\underline{A}_{0}$ term. (b) A cylindrical PDE surface patch.

surface described by the Equation (2) the spine can be constructed by taking that part of the solution with zero mean by way of removing the periodic contribution. One should note that although the particular definition for the spine of a PDE surface that is adopted here is a very convenient one from the point of view of the underlying mathematical representation of the surface, it is by no means a unique definition.

It is noteworthy that the introduction of the $R(u, v)$ term in the new solution described in Equation (6) hardly affect the interior shape of the surface. This is due to the fact that for large $n$ the Fourier modes make negligible contributions to the interior of the patch. Therefore, as far as the spine of the shape generated using Equation (6) is concerned since the spine does not represent the detailed geometry of the shape, the $\underline{A}_{0}(u)$ term is left unchanged by the introduction of the $R(u, v)$ term in the approximate solution and hence the spine of the shape is left unchanged.

To illustrate the idea of generating the spine of a PDE surface, based on the above discussion, the following describes some examples of shapes created using PDE surfaces and the corresponding spines relating to the $\underline{A}_{0}$ term given in Equation (2).

Fig. 2(b) shows a typical PDE surface of cylindrical form. This particular shape is created by means of four appropriately spaced ellipses defining the boundary conditions $\underline{P}_{0}(v), \underline{P}_{1}(v), \underline{d}_{0}(v)$ and $\underline{d}_{1}(v)$. Fig. $2(\mathbf{a})$ shows the image of the cubic polynomial described by the $\underline{A}_{0}$ term corresponding to the spine of this surface patch.

Fig. 3(b) shows a composite shape that looks like the shape of an aircraft. This shape is created by means of five surface patches (one for the fuselage and two for each wing) with common boundaries where necessary. The corresponding composite spine for the aircraft shape is shown is Fig. $3(\mathbf{a})$. As can be noted, in both these examples the spine closely describes the centreline of the object. 


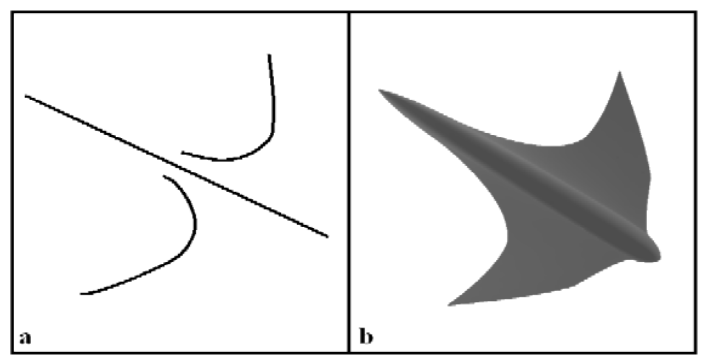

Figure 3: Description of the spine of a composite shape. (a) The corresponding composite spine for the aircraft shape. (b) The shape of an aircraft created by means of five separate PDE surface patches

\section{Shape Parameterisation using the Spine}

One of the many attractive features of PDE surfaces is their ability to be able to create and manipulate complex shapes with ease. Previous work on interactive design has demonstrated that a user with little or no knowledge of solving PDEs and how the boundary conditions affect the solutions of the PDEs is able to use the method to create complex geometry with ease $[11,12,5]$. The shapes in this case are parameterised using the boundary curves that define them. However, it was noted that for very complex shapes, in order to manipulate the shape, the number of curve manipulations can be excessive. Since the spine of the surface characterises the surface patch as a whole, rather than individual boundaries, a shape parameterisation based on the spine would provide further intuitive tools for shape manipulation.

The aim of this section is to show that the spine of a PDE surface can be used to parameterise the surface shape which in turn can be utilised to develop design tools for further efficient shape manipulation. As shown in the previous section the spine of a PDE surface comes as a by-product of the analytic solution used. By virtue of the very definition of the spine it can be seen as a powerful and intuitive mechanism to manipulate the shape of surface once it is defined. There are many ways by which one could utilise the spine to parameterise a PDE surface. One such possibility is described here.

Consider the cubic polynomial described by the $\underline{A}_{0}$ term in Equation (2) to be a Hermite curve of the form,

$$
\underline{H}(u)=\underline{B}_{1}(u) \underline{p}_{1}+\underline{B}_{2}(u) \underline{p}_{2}+\underline{B}_{3}(u) \underline{v}_{1}+\underline{B}_{4}(u) \underline{v}_{2},
$$

where the $\underline{B}_{i}$ are the Hermite basis functions, the vectors $\underline{p}_{1}, \underline{p}_{2}$ and $\underline{v}_{1}, \underline{v}_{2}$ define the position and the speed of the Hermite curve at $u=0$ and $u=1$ respectively. By comparing the Hermite curve given in Equation (10) with 


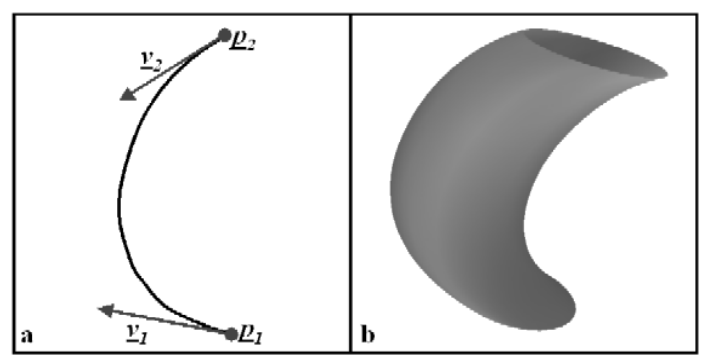

Figure 4: Shape parameterisation and manipulation using the spine. (a) The spine described by the $\underline{A}_{0}$ term defined as a Hermite curve. (b) A deformed cylinderical PDE surface.

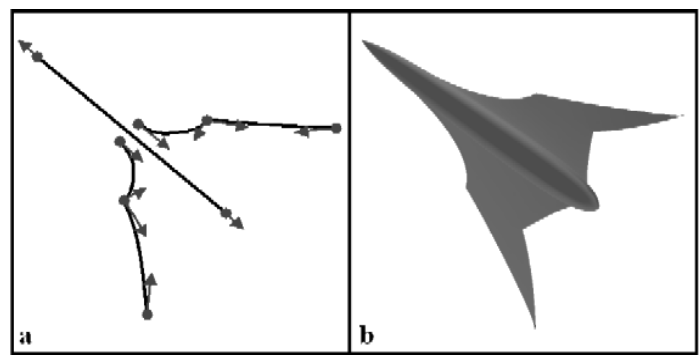

Figure 5: Shape parameterisation and manipulation using the spine. (a) The composite spine described by the corresponding $\underline{A}_{0}$ terms defined as Hermite curves. (b) Manipulated shape of the aircraft shape.

the cubic for the spine given as the $\underline{A}_{0}$ term in Equation (2), the vector constants $\underline{a}_{00}, \underline{a}_{01}, \underline{a}_{02}$ and $\underline{a}_{03}$ given in Equation (3) can be related to the position vectors and its derivatives at the end points of the spine. Since the $\underline{A}_{0}$ term in Equation (2) is an integral part of the solution that generates the surface shape any change in the shape of the spine will of course have a resulting change in the shape of the surface. A useful mechanism to change the shape of the spine would be to manipulate its position and the derivative at the two end points. Therefore, the position vectors and its derivatives at the end of points of the Hermite curve describing the spine are defined to be a new set of shape parameters that enable to manipulate the shape in an intuitive fashion.

To demonstrate this idea, consider the cylindrical shape shown in Fig. 2(b) where the corresponding spine is also shown in 2(a). Fig. 4(b) shows a resulting shape after some manipulation of the shape shown in Fig. 2(b) using the shape parameters defined on the spine. Fig. 4(a) illustrates the resulting spine and the parameters $\underline{p}_{1}, \underline{p}_{2}$ and $\underline{v}_{1}, \underline{v}_{2}$ for the resulting surface patch. 
Fig. 5(b) shows another example of shape manipulation using the spine. Here the shape of the aircraft shown in Fig. 3(b) was manipulated using the parameters defined on the composite spine shown in Fig. 3(a). The final shape of the composite spine and the parameters corresponding to the resulting shape of the aircraft are shown in Fig. 5(a)

Note in both these examples the shape manipulations were carried out interactively via the position vectors $\underline{p}_{1}$ and $\underline{p}_{2}$ and the direction vectors $\underline{v}_{1}$ and $\underline{v}_{2}$, for each of the corresponding surface patch. In the case of the position vectors they are changed by means of clicking and dragging the points in 3space and in the case of direction vectors they are changed by means of the changing the size and the direction of the arrows shown.

\section{$4 \quad$ Higher Order PDE Surfaces}

This section focuses on how the previous discussions on the spine based shape parameterisation can be extended to higher order PDE surfaces. Higher order PDE surfaces posses extra degrees of freedom and therefore are capable of generating more complex surface patches. Furthermore, due to the extra boundary conditions that can be associated with the surface patch, there is a greater level of user control on such a surface patch.

For the sake of keeping the discussion on higher order surfaces concise the PDE chosen here is the tri-harmonic PDE,

$$
\left(\frac{\partial^{2}}{\partial u^{2}}+a^{2} \frac{\partial^{2}}{\partial v^{2}}\right)^{3} \underline{X}(u, v)=0,
$$

which posses higher order than the widely used bi-harmonic form of the PDE given in Equation (1).

In order to cater for the added boundary conditions the tri-harmonic PDE requires extra curvature boundary conditions. For a given surface patch these conditions are defined as $c_{1}$ and $c_{2}$ at $u=0$ and $u=1$ respectively. Fig. 6(a) illustrates how the new boundary conditions can be defined. Following the approach described in Section 2, for defining the boundary conditions using space curves, the curvature boundary conditions, $\frac{\partial^{2} X}{\partial u^{2}}$, are defined by means of the curves marked $P_{1}$ and $P_{2}, d_{1}$ and $d_{2}$ and those marked $c_{1}$ and $c_{2}$ such that $\frac{\partial^{2} \underline{X}}{\partial u^{2}}=[\underline{p}(v)-2 \underline{d}(v)+\underline{c}(v)] t$, where $t$ is a scalar.

Using the approximate analytic solution approach outlined in Section 2.1 the solution of Equation (11) can be written as,

$$
\underline{X}(u, v) \simeq \underline{A}_{0}(u)+\sum_{n=1}^{N}\left[\underline{A}_{n}(u) \cos (n v)+\underline{B}_{n}(u) \sin (n v)\right]+\underline{R}(u, v),
$$




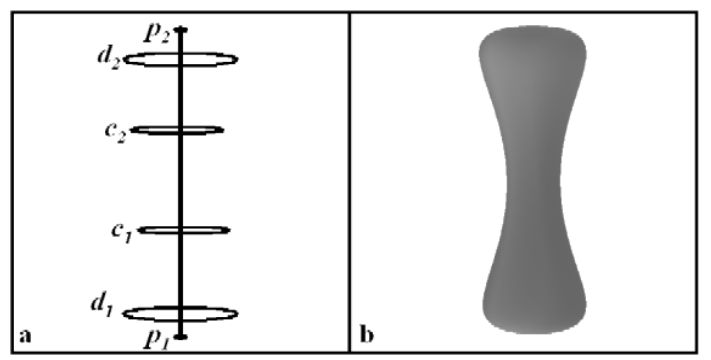

Figure 6: A vesicle shape created using a sixth order PDE surface patch. (a) The boundary conditions and the spine of the vesicle. (b) The shape of the vesicle.

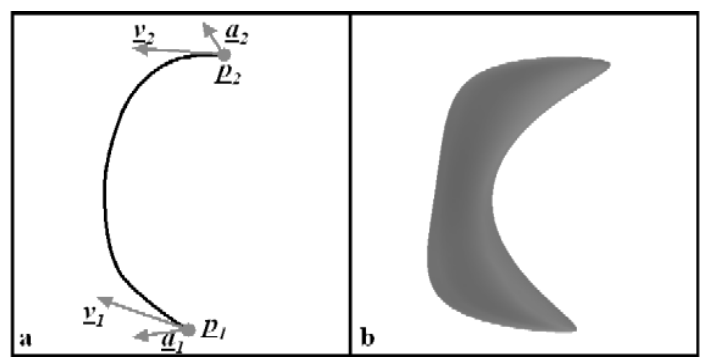

Figure 7: Shape manipulation on the vesicle shape. (a) The spine of the vesicle and the shape parameters defined on the Hermite curve. (b) The resulting shape of the vesicle.

where

$$
\underline{A}_{0}=\underline{a}_{00}+\underline{a}_{01} u+\underline{a}_{02} u^{2}+\underline{a}_{03} u^{3}+\underline{a}_{04} u^{4}+\underline{a}_{05} u^{5},
$$

with $\underline{a}_{04}$ and $\underline{a}_{05}$ being vector valued constants. The terms $\underline{A}_{n}, \underline{B}_{n}$ and $\underline{R}(u, v)$ take similar forms given in Equations (4), (5) and (7) respectively.

Fig. 6(b) shows the shape of a biological vesicle created using a single PDE surface patch based on Equation (11). As discussed earlier the periodic curves shown in Fig. 6(a) are chosen as the boundary conditions for the vesicle shape. Fig. 6(a) also shows the spine for this higher order surface patch described by the $\underline{A}_{0}$ term given in Equation (13).

\subsection{Spine Based Shape Parameterisation}

Taking the approach of using Hermite curves described in Section 3 the surface shape defined by the higher order PDE can also be parameterised using the corresponding spine. An important point to note here is that the $\underline{A}_{0}$ term given in Equation (13) is now a quintic polynomial with two added degrees of control to accommodate the curvatures. Thus, considering this polynomial 


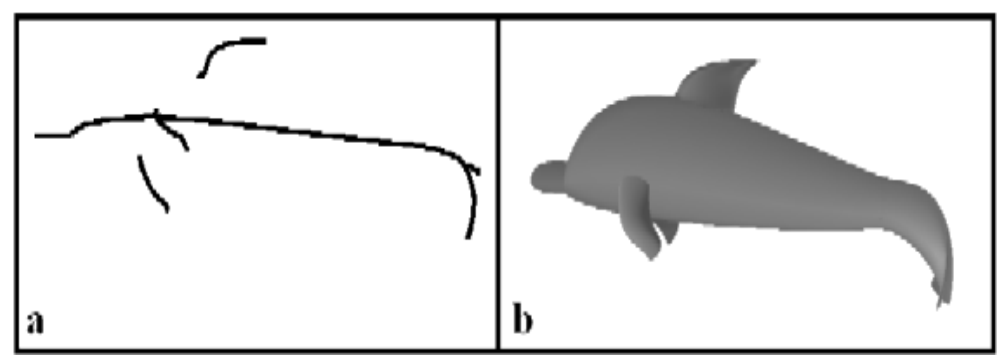

Figure 8: Shape of a dolphin created using a mixture of tri-harmonic and bi-harmonic PDEs. (a) The composite spine of the dolphin shape. (b) The dolphin shape.

to be a Hermite curve of the form,

$$
\underline{H}(u)=\underline{B}_{1}(u) \underline{p}_{1}+\underline{B}_{2}(u) \underline{p}_{2}+\underline{B}_{3}(u) \underline{v}_{1}+\underline{B}_{4}(u) \underline{v}_{2}+\underline{B}_{5}(u) \underline{a}_{1}+\underline{B}_{6}(u) \underline{a}_{2},
$$

where the $\underline{B}_{i}$ are the Hermite basis functions, the vectors $\underline{p}_{1}, \underline{p}_{2}$ and $\underline{v}_{1}, \underline{v}_{2}$ define the position and the speed of the Hermite curve and $\underline{a}_{1}, \underline{a}_{2}$ define the curvature of the curve at the end points of the spine. Therefore, the vectors $\underline{a}_{1}, \underline{a}_{2}$ define an extra set of parameters that can be used to manipulate the shape.

In order to illustrate the shape manipulation using the higher order spine, consider the vesicle shapes shown in Figs. 6(b) and $7(\mathbf{b})$ where the shape manipulation between the two shapes is carried out using the above defined shape parameters on the spine described by the Hermite curve. Fig 7(a) shows the resulting shape of the spine with the shape parameters, $\underline{p}_{1}, \underline{p}_{2}$, $\underline{v}_{1}, \underline{v}_{2}$ and $\underline{a}_{1}, \underline{a}_{2}$ introduced via the Hermite curve. Again the shape manipulation is carried out interactively by means of changing the position and the direction of the vectors defining the shape parameters.

Figs. 8 and 9 further illustrates the use of shape parameters on the spine for use in interactive shape manipulation. Here the shapes of the dolphins are created using a mixture of tri-harmonic and bi-harmonic PDEs. In particular, the main body of the dolphin is created as a single sixth order PDE surface patch. The shape manipulation between the two shapes were carried out using the shape parameters, $\underline{p}_{1}, \underline{p}_{2}, \underline{v}_{1}, \underline{v}_{2}$ and $\underline{a}_{1}, \underline{a}_{2}$, defined on the spine corresponding to the main body of the dolphin shape.

\section{Conclusions}

This paper describes how the spine of a PDE surface can be used to parameterise the shape of a complex object. Due to the analytic form of the solution 


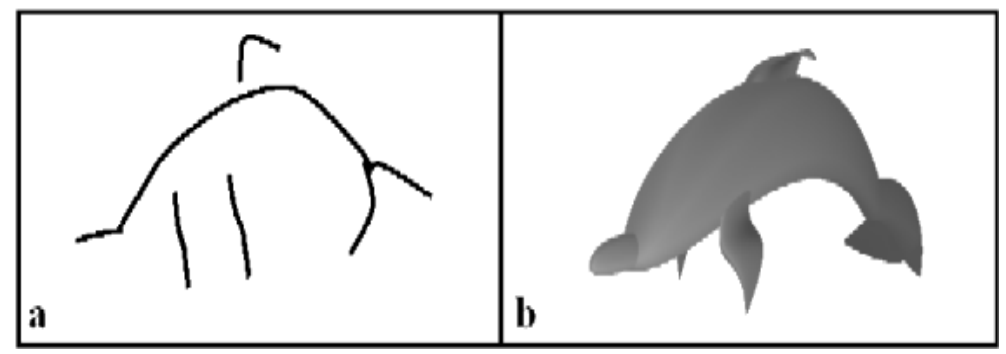

Figure 9: Manipulated shape of the dolphin. (a) The resulting composite spine of the dolphin shape. (b) The resulting dolphin shape.

used to generate the surface shape the spine is computed as a by-product of the solution. It is shown that due to the canonical and intuitive nature of the spine it can be used to parameterise a complex shape. This parameterisation can then be used to manipulate the shape in real time once it is defined.

As shown here the shape manipulation using the spine can be seen as an added bonus to the existing intuitive tools available for efficient shape manipulation of PDE surfaces. Essentially the spine based shape parameterisation undertaken here is formulated using Hermite curves. With this formulation it has been shown that the shape can be efficiently parameterised.

Here we have discussed how the spine can be used to deform a PDE surface globally. Should local control of shape is required this methodology can easily be incorporated within a framework where the relevant PDE for local control is solved (e.g. see [5]). In the examples discussed in this paper the boundary conditions were taken to be curves in 3-space and the spine is interactively manipulated to bring about real time changes in the shape. The interactive design process enables the user to detect and avoid collision of PDE surfaces when the object consists of multiple PDE patches. However, should the manipulation procedure requires to be automatic, for example in the case of automatic design optimisation, then a robust collision detection procedure would have to be implemented in order to avoid collision of adjacent surface patches during the deformation process.

An interesting future direction of study would be to extend the spine based parameterisation to cater for geometry that can handle not only more complex shapes but also shapes with changing topology. Such a parameterisation scheme can then, for example, be applied to design optimisation problems where a wide variety of geometry with changing topology would be available to the optimisation scheme. 


\section{References}

[1] Bloor, M.I.G., and Wilson, M.J.: Spectral Approximations to PDE Surfaces. Computer-Aided Design, 28 (1996) 145-152

[2] Bloor, M.I.G. and Wilson, M. J.: Method for Efficient Shape Parametrization of Fluid Membranes and Vesicles. Physical Review E, 61(4) (2000) 4218-4229

[3] Blum, H.: A transformation for Extracting New Descriptors of Shape. In: Wathen-Dunn, W. (ed.): Models for Perception of Speech and Visual Form, MIT Press (1976) 362-381

[4] Boissonnat, J. D.: Geometric Surfaces for 3-Dimensional Shape Representation. ACM Transactions on Graphics, 3(4) (1984) 244-265

[5] Du, H., and Qin, H.; Direct Manipulation and Interactive Sculpting of PDE Surfaces. Computer Graphics Forum (Proceedings of Eurographics 2000), 19(3) (2000) 261-270

[6] Zhang, J.J and You, L., PDE Based Surface Representation -Vase Design, Computers and Graphics, 26(1) (2002) 89-98

[7] Dutta, D and Hoffmann, C.M.: On the Skeleton of Simple CSG Objecs. ASME Journal of Mechanical Design, 115(1) (1992) 87-94

[8] Farin, G.,: Curves and Surfaces for Computer aided Geometric Design: A Practical Guide. Academic Press, (1990).

[9] Nackman, L.R., and Pizer, S.M.: Three-Dimensional Shape Description using Symmetric Axis Transform. IEEE Transactions on Pattern Analysis and Machine Intelligence, $7(2)$ (1985) 187-202

[10] Patrikalakis, N.M., and Gursoy, H.N.: Shape Interrogation by Medial Axis Transform. In: Ravani, B. (ed.): Advances in Design Automation: Computer-Aided Computational Design, ASME 1 (1990) 77-88

[11] Ugail, H., Bloor, M.I.G., and Wilson, M.J.: Techniques for Interactive Design Using the PDE Method. ACM Transactions on Graphics, 18(2) (1999) 195-212

[12] Ugail, H., Bloor, M.I.G., and Wilson, M.J.: Manipulations of PDE Surfaces Using an Interactively Defined Parameterisation. Computers and Graphics, 24(3) (1999) 525-534 